\title{
Avaliação histopatológica de órgãos reprodutivos e bexiga de fêmeas suínas descartadas
}

\author{
[Histopathology of reproductive organs and bladder of culled sows] \\ G.A. Ritterbusch ${ }^{1}$, C. Sá Rocha ${ }^{1}$, J.R. Ciacci-Zanella ${ }^{2}$, A.L. Amaral ${ }^{2}$, A. Coldebella ${ }^{2}$, \\ K.R. Ascoli ${ }^{3}$, N. Mores $^{2} *$ \\ ${ }^{1}$ Aluno de pós-graduação - Universidade do Estado de Santa Catarina - Lages, SC \\ ${ }^{2}$ Embrapa Suínos e Aves - Concórdia, SC \\ ${ }^{3}$ Centro de Diagnóstico em Sanidade Animal - CEDISA - Concórdia, SC
}

\begin{abstract}
RESUMO
Em um sistema intensivo de produção de suínos, as falhas reprodutivas são uma das principais razões de descarte de matrizes e queda nos índices produtivos. A infecção urinária (cistite) e as endometrites são consideradas importantes causas de descarte em fêmeas suínas, por terem consequências reprodutivas relevantes e elevarem a taxa de reposição do plantel. O presente estudo teve o objetivo de avaliar o aparelho reprodutivo e a bexiga de fêmeas suínas de descarte normal de granjas, bem como investigar a existência de relação entre as patologias encontradas. Foram examinadas 79 matrizes suínas oriundas de 20 rebanhos localizados no Estado de Santa Catarina. De cada fêmea foram coletados os ovários, fragmentos de útero e bexiga. Dentre as fêmeas avaliadas, 32 (40,5\%) tinham diferentes graduações de cistite, $24(30,4 \%)$ tinham algum tipo de inflamação uterina, e $9(11,4 \%)$ estavam em anestro, com ovários inativos. Contudo, não foi observada dependência significativa entre cistite e endometrite nas amostras analisadas.
\end{abstract}

Palavras-chave: porca, cistite, endometrite, anestro, descarte

\begin{abstract}
Reproductive failures are the major reasons for removal of sows and decrease of production rates in an intensive swine production system. Urinary infection and endometritis are considered important causes for culling of sows, due to relevant reproductive consequences and increase of the replacement rates. The present study aimed to evaluate the reproductive and urinary system of culled sows, as well as investigate the occurrence of cystitis and endometritis in analyzed sows. Samples, such as ovaries, uterus fragments and bladder were collected from 79 sows originated from 20 farms of Santa Catarina State. Results showed that, 32 (40,5\%) analyzed sows presented cystitis in different levels, 24 (30,4\%) had some class of uterine inflammation, and $9(11,4 \%)$ were in anestrous, with inactive ovaries. However, unsignificative dependence between cystitis and endometritis in analyzed samples was observed.
\end{abstract}

Keywords: sows, cystitis, endometritis, anestrous, culling

\section{INTRODUÇÃO}

As infecções do aparelho genitourinário estão entre as patologias mais importantes da fêmea suína e podem se manifestar nas diferentes fases do ciclo de produção. Os prejuízos causados por essas patologias envolvem, principalmente, falhas reprodutivas - influenciando negativamente a produtividade do rebanho -, problemas na saúde geral das matrizes e consequente aumento nas taxas de mortalidade de porcas e de reposição (Sobestiansky et al., 1995; Almond et al., 2006; Drolet e Dee, 2006).

Recebido em 28 de março de 2013

Aceito em 8 de agosto de 2013

*Autor para correspondência (corresponding author)

E-mail: nelson.mores@embrapa.br 
As falhas reprodutivas em decorrência de infecções no aparelho genitourinário geralmente apresentam-se com diminuição no tamanho de leitegadas, aumento da ocorrência de descargas vulvares, retorno ao cio, e com quadros de mastite - metrite - agalaxia (MMA), além de estar relacionada com casos de morte súbita de fêmeas nas fases de gestação e lactação (Dalla Costa e Sobestiansky, 1999).

Segundo Wentz (1986), as infecções genitais podem ocorrer juntamente com infecções urinárias ou serem consequência delas, sendo a má higiene, principalmente nos locais onde as porcas habitualmente se sentam, um fator que promove alta pressão infectiva ambiental, possibilitando maior ocorrência desse tipo de infecção. Além disso, estudos relatam a existência de estreita relação entre as infecções urinárias e os problemas reprodutivos em matrizes em produção (Waller et al., 2002; Silveira et al., 2006).

Nesse contexto, o presente estudo foi desenvolvido com o objetivo de verificar a frequência de alterações patológicas na bexiga e nos órgãos reprodutivos de matrizes suínas descartadas sem causa definida.

\section{MATERIAL E MÉTODOS}

Durante os meses de setembro a dezembro de 2008, foram analisadas 79 porcas em quatro abatedouros da região oeste e meio oeste de Santa Catarina, oriundas de 20 rebanhos suínos (três a cinco porcas por rebanho). Esses animais foram amostrados de forma aleatória nos abatedouros, sem direcionar a razão específica de seus descartes dos rebanhos. De cada porca foram colhidos um fragmento de cada corno uterino (região média), ovários e um fragmento de bexiga. Esses tecidos foram fixados em formol $10 \%$ tamponado, processados pelo método de inclusão em parafina, seccionados a $5 \mu$ e corados com hematoxilina e eosina (Luna, 1968).

Os tecidos foram observados ao microscópio óptico para verificação de alterações teciduais. De acordo com a gravidade e tipo das alterações histológicas, a bexiga foi classificada em: a) normal (sem alterações histológicas), b) cistite leve (discreta infiltração mononuclear ou mista na mucosa e submucosa), c) cistite moderada (lesões inflamatórias mais acentuadas que a anterior e discreta hiperplasia do epitélio) e d) cistite severa (acentuada infiltração inflamatória, edema e hiperemia/hemorragia na mucosa e submucosa e com hiperplasia epitelial). Para o tecido uterino, foi utilizado o mesmo procedimento de avaliação histológica e classificado em: a) normal (sem alterações histológicas), b) endometrite catarral (discreta infiltração inflamatória mista ou com predomínio de mononucleares no endométrio e/ou no lúmen das glândulas/órgão), c) endometrite purulenta (acentuada infiltração e exsudação de neutrófilos no endométrio e/ou no lúmen das glândulas/órgão) e d) endometrite crônica (infiltração inflamatória mononuclear e fibroplasia ao redor de glândulas endometriais e/ou na lâmina própria/epitélio). Já os ovários foram avaliados quanto ao período do ciclo estral das fêmeas, classificando-os em: a) ciclando, b) em anestro ou c) com ovário cístico, segundo a metodologia descrita por Carvalho (1990). Análises estatísticas foram realizadas utilizando o teste de $\chi^{2}$ a fim de verificar a existência de dependência entre a ocorrência de cistite e a ocorrência de endometrite.

\section{RESULTADOS E DISCUSSÃO}

Os resultados do exame histopatológico de bexiga, útero e ovário das 79 fêmeas são apresentados na Tabela 1. Dos fragmentos de bexiga analisados, $47(59,49 \%)$ amostras encontravam-se normais e $32(40,51 \%)$ tinham diferentes graduações de cistite (Fig. 1). Em estudo semelhante, Pôrto et al. (2004) observaram lesões de cistite em $25 \%$ das amostras avaliadas.

No exame do útero, $55(69,62 \%)$ estavam normais e $24(30,38 \%)$ tinham algum tipo de endometrite (Fig. 2).

Ao analisar os cortes histológicos dos ovários, verificou-se que $86,08 \%$ das fêmeas encontravam-se ciclando (presença de folículos em crescimento, corpos lúteos ou corpos hemorrágicos), $2,53 \%$ tinham cistos ovarianos e $11,39 \%$ encontrava-se em anestro, com ovários inativos. 

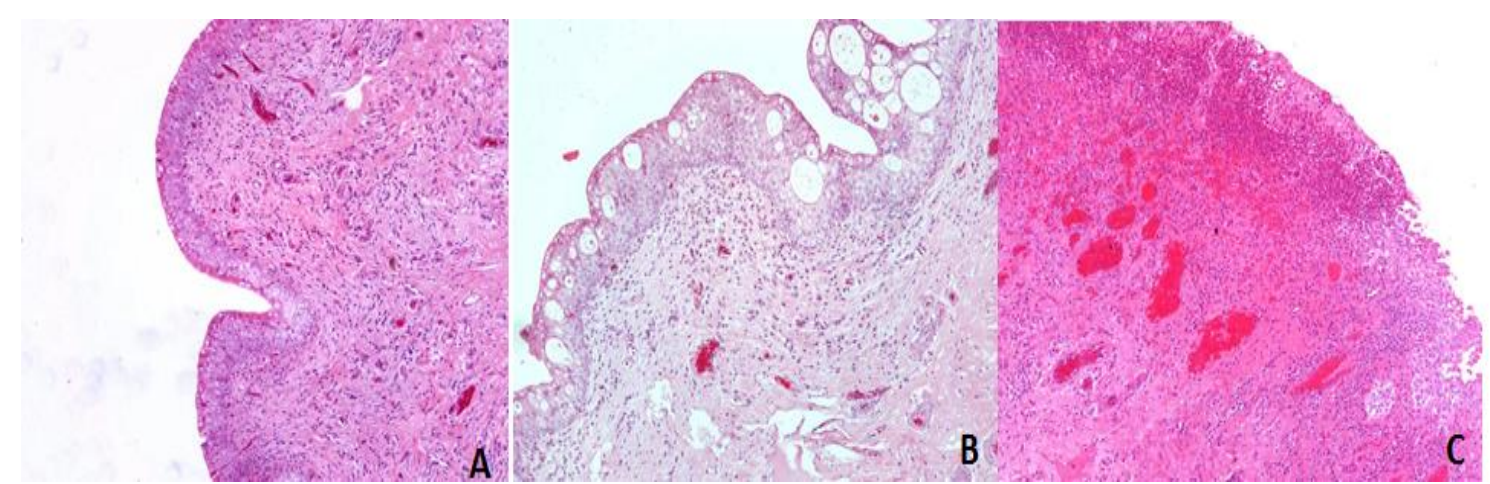

Figura 1. Lesões observadas no exame histopatológico de bexiga: A - cistite leve; B - cistite moderada; C - cistite severa. Hematoxilina-eosina (Objetiva 10x).
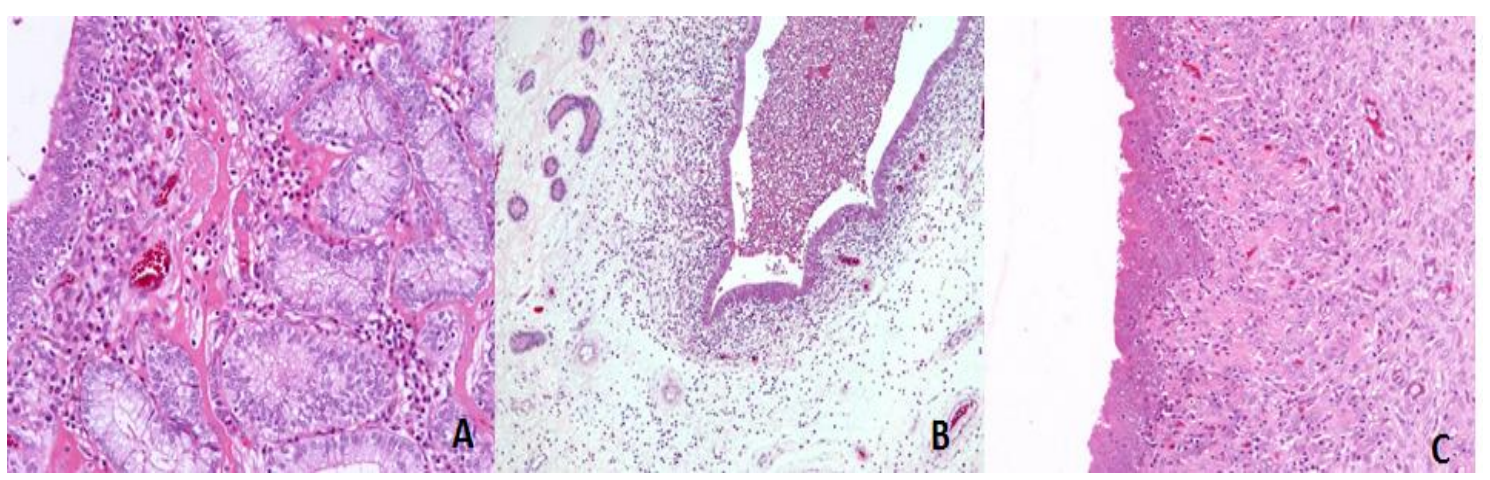

Figura 2. Lesões observadas no exame histopatológico de útero: A - endometrite catarral (Objetiva 20x); B - endometrite purulenta (Objetiva 10x); C - endometrite crônica (Objetiva 10x). Hematoxilina-eosina.

Tabela 1. Resultados do exame histopatológico de bexiga, útero e ovário das 79 porcas descartadas

\begin{tabular}{|c|c|c|c|c|}
\hline \multirow{2}{*}{ Exame histopatológico } & \multicolumn{4}{|c|}{ Frequência } \\
\hline & $\mathrm{N}^{\circ}$ & Total $\mathrm{N}^{\circ}$ & $\%$ & Total, \% \\
\hline Cistite leve & 15 & & 18,99 & \\
\hline Cistite moderada & 14 & 32 & 17,72 & 40,51 \\
\hline Cistite severa & 3 & & 3,80 & \\
\hline Endometrite catarral & 6 & & 7,60 & \\
\hline Endometrite purulenta & 7 & 24 & 8,86 & 30,38 \\
\hline Endometrite crônica & 11 & & 13,92 & \\
\hline Ovário ciclando & 68 & 68 & 86,08 & \\
\hline Ovário cístico & 2 & 11 & 2,53 & 100,00 \\
\hline Ovário em anestro & 9 & 11 & 11,39 & \\
\hline
\end{tabular}

$\mathrm{Na}$ análise estatística, observou-se que não houve dependência significativa $(\mathrm{P}>0,05)$ entre cistite $\mathrm{e}$ endometrite nas fêmeas avaliadas (Tab. 2), diferindo de resultados obtidos em outros trabalhos. Waller et al. (2002) verificaram que fêmeas com problemas urinários tinham 8,9 vezes mais risco de apresentar problemas patológicos no trato genital. Em outro relato, Buzato et al. (2006) observaram que porcas com problemas urinários na entrada da maternidade apresentaram oito vezes mais risco de ocorrência de problemas com doenças do periparto. Da mesma forma, porcas afetadas por urocistite apresentaram 3,5 vezes mais probabilidade de ter endometrite simultaneamente (Biksi et al., 2002). Por outro lado, com base em nossos achados, observa-se que todas as fêmeas que estavam em anestro apresentavam endometrite crônica ou purulenta, bem como algum grau de cistite, mostrando clara associação entre essas duas patologias. 
Tabela 2. Relação das lesões de bexigas e úteros das 79 porcas examinadas

\begin{tabular}{|c|c|c|c|c|}
\hline & & \multicolumn{2}{|c|}{ Útero } & \multirow{2}{*}{ Total } \\
\hline & & Normal & Endometrite & \\
\hline \multirow{3}{*}{ Bexiga } & Nomal & $34(43,04)$ & $13(16,46)$ & $47(59,49)$ \\
\hline & Cistite & $21(26,58)$ & $11(13,92)$ & $32(40,51)$ \\
\hline & Total & $55(69,62)$ & $24(30,38)$ & $79(100,00)$ \\
\hline
\end{tabular}

$\mathrm{P}=0,524$ pelo teste qui-quadrado.

No manejo de descartes de fêmeas em um rebanho suíno, as variáveis que afetam a eficiência reprodutiva são as razões mais importantes em que os técnicos se baseiam (Dartora et al., 1997). Os achados no presente trabalho mostram que muitas das porcas que são descartadas do rebanho apresentavam inflamação no aparelho reprodutor e/ou bexiga, o que possivelmente comprometeu a eficiência reprodutiva, motivando seu descarte. Nesse aspecto, considerando a alta frequência de cistite e endometrite encontrada neste trabalho, em porcas de descarte de rotina dos rebanhos, sugere-se que sejam implementadas medidas mais eficazes de controle das infecções que possam afetar a integridade do aparelho genitourinário no plantel de matrizes.

É importante enfatizar que a maioria dos descartes gera a reposição dessas porcas com leitoas. Além do custo de reposição, leitoas primíparas têm baixa imunidade e baixa qualidade de colostro, comprometendo o equilíbrio imunológico do rebanho (Smits, 2011).

Dessa forma, os dados apresentados aqui reforçam a necessidade de os técnicos de granjas de suínos manterem um eficiente programa de monitoramento das porcas para controle dessas infecções nos rebanhos, evitando perdas e reposições desnecessárias.

\section{CONCLUSÃO}

É alta a frequência de cistite e endometrite em porcas normalmente descartadas pelos produtores na região oeste e meio oeste de Santa Catarina. Existe forte relação entre endometrite com anestro, mas não entre cistite com endometrite.

\section{REFERÊNCIAS}

ALMOND et al. Disease of reproductive system. In: STRAW, B.E.; ZIMMERMAN, J.; D'ALLAIRE, S.; TAYLOR, D.J. (Eds.). Diseases of swine. 9.ed. Blackwell Publishing Ames, Iowa, USA, 2006. p.113148.

BIKSI, I.; TACKACS, N.; VETES, F. et al. Association between endometritis and urocystitis in culled sows. Acta Vet. Hung, v.50, p.413-23, 2002.

BUZATO, A.; SILVEIRA, P.R.; AMARAL, A.L. et al. Relação entre infecções urinárias e problemas do periparto em porcas. In: CONGRESSO LATINOAMERICANO DE SUINOCULTURA. 3., 2006, Foz de Iguaçu. Anais... Concórdia: Embrapa Suínos e Aves, 2006. p.943-945.

CARVALHO, L.F.O.S. Investigação clínica, anatomopatológica e citogenética de fêmeas suínas com transtornos reprodutivos. 1990. 95f. Tese (Doutorado em Medicina Veterinária) - Faculdade de Medicina Veterinária e Zootecnia, Botucatu, SP.

DALLA-COSTA, O.A.; SOBESTIANSKY, J. Como controlar a infecção urinária em matrizes suínas em produção. Concórdia: EMBRAPA-CNPSA, 1999. 2p. (Comunicado Técnico, $\mathrm{n}^{\circ} 10$ ).

DARTORA, V.; MORÉS, N.; WOLOSZYN, N. Procedimentos Básicos na Produção de Suínos. BIPERS: Boletim Informativo Pesquisa - Embrapa Suínos e Aves/Extensão - EMATER/RS. Ano 6, 1997, $18 \mathrm{p}$.

DROLET, R.; DEE, S.A. Disease of the urinary system, In: STRAW, B.E.; ZIMMERMAN, J.; D'ALLAIRE, S.; TAYLOR, D.J. (Eds.). Diseases of swine. 9.ed. Blackwell Publishing Ames, Iowa, USA, 2006. p.199-218.

LUNA, L.G. Manual of histologic staining methods of the Armed Forces Institute of Pathology. 3.ed. New York: McGraw Hill Book Company, 1968. 260p.

PÔRTO, R.N.G.; SOBESTIANSKY, J.; MATOS, M.P.C.; MEIRINHOS, M.L.G. Aspectos histopatológicos do sistema urinário de matrizes suínas descartadas. Cienc. Anim. Bras., v.5, p.109-112, 2004. 
SILVEIRA, P.R.; BUZATO, A.M.; CABRAL, H.C. et al. Relação Entre Infecção Urinária e Problemas Puerperais em Porcas. Concórdia:Embrapa Suínos e Aves, 2006. 2p. (Embrapa Suínos e Aves. Comunicado técnico $n^{\circ} 433$ ).

SMITS, R.J. Impact of the sow on progeny productivity and herd feed efficiency. In: Recent Advances in Animal Nutrition - Austrália, v.18, 2011, p.61.

SOBESTIANSKY, J.; PERUZZO, B.F.; DALLA COSTA, O.A.; ALBERTON, G. Infecção urinária na fêmea suína em produção: ocorrência em granjas com queda da eficiência reprodutiva. In: CONGRESSO BRASILEIRO DE VETERINÁRIOS ESPECIALISTAS EM SUÍNOS, 7., 1995. Blumenau. Anais... Blumenau: SC, ABRAVES, 1995. p.68.
WALLER, C.H.; BILKEI, G.; CAMERON, R.D.A. Effect of periparturiente diseases accompanied by excessive vulval discharge and weaning to mating interval in sow reproductive performance. Aust. Vet. J., v.80, p.545-549, 2002.

WENTZ, I.; SILVEIRA, P.R.S.; PIFFER, I.A. et al. As infecções uterinas como causa de repetição de cobrição em porcas. Concórdia: Embrapa Suínos e Aves, 1986. 3p. (Embrapa Suínos e Aves. Comunicado técnico $\mathrm{n}^{\circ} 112$ ). 